\title{
Abaritana ou Avaritana provincia
}

\section{J. Desanges et S. Chaker}

\section{OpenEdition}

Journals

Édition électronique

URL : http://journals.openedition.org/encyclopedieberbere/772

DOI : $10.4000 /$ encyclopedieberbere. 772

ISSN : 2262-7197

\section{Éditeur}

Peeters Publishers

\section{Édition imprimée}

Date de publication : 1 novembre 1984

Pagination : $57-59$

ISBN : 2-85744-201-7

ISSN : 1015-7344

\section{Référence électronique}

J. Desanges et S. Chaker, «Abaritana ou Avaritana provincia », Encyclopédie berbère [En ligne], 1 | 1984, document A6, mis en ligne le 01 décembre 2012, consulté le 05 octobre 2020. URL : http://

journals.openedition.org/encyclopedieberbere/772 ; DOI : https://doi.org/10.4000/ encyclopedieberbere. 772

Ce document a été généré automatiquement le 5 octobre 2020.

(c) Tous droits réservés 


\title{
Abaritana ou Avaritana provincia
}

\author{
J. Desanges et S. Chaker
}

\section{Par J. Desanges}

1 Victor de Vita, I, 13 (dans Mon. Germ. Hist., auct. ant., III/1, éd. C. Halm, Berlin, 1879, p. 4) nous apprend qu'en 442, Geiséric attribua à son armée la Zeugitane ou Proconsulaire, se réservant la Byzacène, l'Abaritana et la Gétulie, ainsi qu'une partie de la Numidie (sibi Byzacenam, Abaritanam atque Getuliam et partem Numidiae reservavit). Cette phrase nous interdit de considérer l'Abaritana comme une partie de la Proconsulaire, de la Byzacène ou de la Numidie (en un sens que la mention de la Gétulie restreint à la Numidie de Cirta peut-être augmentée du nord de la Numidie d'Hippone). L'Abaritana n'est pas non plus la Gétulie, mais l'emploi de la conjonction atque à l'intérieur de l'énumération pourrait la lier plus étroitement à celle-ci. Si l'on en croit la Table de Peutinger, segm. IV, 2-5, la Gétulie commençait au sud (c'est-à-dire en réalité au sud-ouest) d'une ligne Gadiaufala (Ksar Sbahi) -Theveste (Tebessa). Plus à l'ouest, sa limite septentrionale ne devait pas être très au sud de Cirta, d'après des indications de Salluste, Jug., CIII, 4, et du Bell. Afr., XXV, 2 (cf. J. Gascou, Le cognomen Gaetulus, Gaetulicus en Afrique romaine, dans M.E.F.R., LXXXII, 1970, p. 732-734). L'Abaritana pourrait être une partie reculée de la Gétulie, si l'on prend en considération un autre témoignage qu'on peut dater de la décennie 445/455, soit une trentaine d'années avant la rédaction de l'Historia persecutionis de Victor de Vita, celui du Liber Promissionum, III, 45 (dans Opera Quoduultdeo Carthaginiensi episcopo tributa, éd. R. Braun. Corpus Christ., series Latina, LX, Turnholt, 1976, p. 186). L'auteur de ce livre, sans doute Quoduultdeus de Carthage, en vient à dire : " J'ai vu moi-même aussi dans un coin de la province avaritaine (in quadam parte Avaritanae provinciae), tirer de grottes et de cavernes d'antiques idoles qui y avaient été cachées, de sorte que toute cette ville (omnis illa... civitas) avec son clergé était sous le coup d'un parjure sacrilège ". Il apparaît donc que le christianisme a été tardivement introduit dans cette province et qu'en tout cas, le paganisme était encore dominant au début du $\mathrm{v}^{\mathrm{e}}$ siècle (cf. R. Braun, Un témoignage littéraire méconnu sur l' Abaritana provincia, dans Rev. Afr., CIII, 1959, p. 116); d'autre part, la mention d'une 
civitas, comme l'a déjà remarqué R. Braun, suggère fortement que la dite province a tiré son nom de celui d'une ville.

D'autres mentions vraies ou supposées de l'adjectif ethnique sont à prendre en considération. Y a-t-il beaucoup à tirer de l'indication de Pline l'Ancien, H.N., XVI, 172, selon laquelle « le roseau le plus renommé pour fabriquer des ustensiles de pêche est le roseau abaritain en Afrique" ? Contrairement à Chr. Courtois (Victor de Vita et son œuvre, Alger, 1954, p. 36, n. 101), nous ne croyons pas que la mention de roseaux nous impose de localiser l'Abaritana provincia à proximité de la mer, au delà de la Byzacène. Ces roseaux pouvaient en effet provenir d'une région de l'intérieur. Quant à l'existence, souvent affirmée, d'un évêché Abaritanus en Proconsulaire (cf. J. Mesnage, L'Afrique chrétienne, Paris, 1912, p. 174), il est permis d'en douter. En effet, on est bien tenté de penser que Félix Abaritanus, mentionné en second parmi les évêques de Proconsulaire et signalé en exil par la Notitia provinciarum et civitatum Africae (dans M.G.H. a. a., III/1, p. 63), ne fait qu'un avec Félix Abbiritanus exilé par Hunéric à la même époque, à en croire Victor de Vita, II, 26 (ibid., p. 19). Il serait tout à fait curieux que Félix Abaritanus et Félix Abbiritanus ayant été tous les deux exilés par Hunéric à la même époque, un seul d'entre eux fût mentionné dans la Notitia, et seul l'autre par Victor de Vita. On s'accorde donc à croire qu'il n'y a eu qu'un seul évêque en cause, Félix Abbiritanus (Chr. Courtois, ibid., p. 46, n. 159; A. Mandouze, Prosopographie de l'Afrique chrétienne, Paris, 1982, art. Felix 61, p. 432-433). Il faut à notre avis, rayer le siège épiscopal d'une hypothétique Abaris de Proconsulaire qu'aucun autre témoignage n'atteste. En tout état de cause, nous savons que l'Abaritana est opposée à la Proconsulaire par Victor de Vita. L. Schmidt (Geschichte der Wandalen, Leipzig, 1942², p. 71) a voulu localiser l'Abaritana en Maurétanie «Gaditaine », parce que, selon le Géographe de Ravenne (I, 3 ; III, 11 deux fois), cette région (qui est celle de Ceuta) était appelée par les indigènes Abrida. Mais Chr. Courtois, op. l, p. 36, n. 101, a fait remarquer que le mot abrida est un nom commun qui signifie dans certains dialectes berbères " passage ». Or, à supposer qu'il y ait un rapport effectif entre Abaritana et abrida, plus d'une contrée pourrait être qualifiée de " passage ». Quelle que soit l'aptitude particulière de la bordure africaine du détroit de Gibraltar à recevoir un tel surnom, l'hypothèse de L. Schmidt est inacceptable, car Geiséric ne s'est assurément pas réservé la lointaine région du détroit de Gibraltar, alors qu'il ne possédait ni la Sitifienne, ni la Césarienne. A juste titre donc, Chr. Courtois a considéré le problème comme toujours ouvert. Il a proposé (ibid.) de localiser l' Abaritana provincia au sud de Mareth, dans la partie côtière de la Tripolitaine (au sens antique) occidentale. Mais le Liber promissionum mentionne le pays comme encore très païen au début du ve siècle ; or Girba (dans l'île de Djerba, voisine de Mareth) possédait un évêque depuis au moins 256 de notre ère (cf. J. Mesnage, L'Afrique chrétienne, p. 56 ; J.L. Maier, L'épiscopat de l'Afrique romaine, vandale et byzantine, Rome-Neuchâtel, 1973, p. 147 et 365-366). D'autre part et surtout, pour Victor de Vita (III, 42-45, op. cit., p. 51) la Tamallumensis civitas, en quoi il faut reconnaître Turris Tamalleni (Telmine) à l'est du Djerid, était dans le voisinage de la Tripolitaine. Il est dès lors difficile d'admettre que l' Abaritana ait été mentionnée par Victor de Vita dans le partage du royaume de Geiséric, sans que le fût la Tripolitaine, à laquelle elle se fût en quelque sorte substituée.

Une stèle inscrite de Timgad, très sommairement publiée en 1911 par A. Ballu (B.A.C., 1911, p. 131) et sur laquelle J. Gascou et M. Janon ont aimablement attiré notre attention, mentionne un couple résidant in vicu $(=$ in vico) Abaris (toponyme non décliné). L'inscription peut être datée, par la paléographie et surtout par la coiffure de 
la femme représentée sur la stèle, de la fin du second siècle de notre ère (cf. J. Desanges, Le uicus Abaris et l'Abaritana prouincia, dans B.A.C., 1981 B, à paraître). On constate donc qu'il y avait à cette époque un vicus Abaris dans la région de Timgad, comme il y avait un vicus Aureli sur la voie de Thamugadi (Timgad) à Theveste (Tebessa) d'après la Table de Peutinger (segm. III, 5). Cette inscription conforte quelque peu l'hypothèse formulée jadis (cf. Byzantion, XXXIII, 1963, p. 54-55) que l'Abaritana est la partie de la Gétulie qui correspond au massif de l'Aurès. On sait que ce massif fut occupé par les Vandales peu après leur arrivée en Afrique selon Procope (De Aed., VI, 7, 6). Par la suite, ils en furent chassés par les Maures. On peut supposer que le vicus Abaris devenu civitas donna à cette époque son nom à toute la région que les Vandales nommèrent Abaritana provincia, de façon quelque peu abusive, mais qui étonnera moins si l'on considère qu'en 419, l'Eglise d'Afrique évoquait une Arzugitana provincia confondue avec la Byzacène (cf. infra, art. Arzuges). On remarquera qu'Ibn Khurradâdh-bih (Description du Maghreb et de l'Europe au III = IXe siècle, éd. M. Hadj Sadok, Alger, 1949, p. 11) nomme Awāris le massif de l'Aurès qu'un texte parallèle d'Ibn al-Faqīh al-Hamadhānī (ibid., p. 33) nomme Awrās, forme courante de l'oronyme chez les géographes arabes. S'il ne s'agit pas là d'une opposition à valeur morphologique propre à la langue arabe (Awāris, corrigé en Awārīs, pourrait être un "pluriel de pluriel " par rapport à Awrās), on serait tenté de rapprocher Awāris d'Avaritana (Liber promissionum) et Awrās du surnom ou sobriquet Aurasius (C.I.L., VIII, 2476, à $16 \mathrm{~km}$ au sud-est de Lambèse), Aurassius (C.I.L., 2848, Lambèse) ou Aurasus (C.I.L., 2626, a, 16, Lambèse, sous l'empereur Valérien) et de l'oronyme, attesté seulement au $\mathrm{V}^{\mathrm{e}}$ siècle, Aúpóciov (Procope, passim) et, sous forme d'adjectif, Aurasitana (Corippus, Joh., II, 149).

\section{Abaritana/Abaris $\leftrightarrow$ Awaris/Awras ? (S. Chaker)}

5 L'hypothèse évoquée par J. DESANGES d'un lien entre Abaritana/Abaris et Awaris/Awras (Aurès) n'est pas incompatible avec les correspondances phonétiques que l'on peut entrevoir entre libyque et berbère moderne.

6 Certains indices permettent en effet d'envisager une correspondance partielle libyque / $\mathrm{b} /$ (ou /b/, noté « $\mathrm{b} »$ en latin ?) $\leftrightarrow$ berbère /w/-/u/, notament en position implosive :

LIBYQUE « BERBÈRE »

TBGG *TWGGA $\rightarrow$ Tugga

$\mathrm{RNB}$ « vaincre » $* \mathrm{RNW} \rightarrow r n u$

GZB *GZW $\rightarrow$ agez, « garder »

7 La forme latine Micipsa (libyque MKWSN) est probablement un témoignage complémentaire de cette correspondance et de son caractère partiel : certaines formes du libyque avaient (déjà) /w/ en face de $/ \mathrm{b} / \mathrm{-} / \mathrm{b} /$.

8 Ce type de correspondances est du reste encore bien attesté à l'heure actuelle entre le parler de Ghadames $(/ b /)$ et le reste du berbère $(/ \mathrm{w} /-/ \mathrm{u} /)$ :

GHADAMES « BERBÈRE »

$r n e B$ « vaincre » rnu 
INDEX

Mots-clés : Algérie, Linguistique, Histoire, Onomastique 